\title{
INSECTICIDE EFFICACY OF SPATIAL REPELLENT COMPOUND-METOFLUTHRIN AGAINST SUSCEPTIBLE AND RESISTANT STRAINS OF AEDES AEGYPTI
}

\author{
EMAD I. M. KHATER ${ }^{\prime}$, DING ZHU ${ }^{2}$, CHRISTOPHER S. BIBBS $^{3}$, \\ AND RUI-DE XUE ${ }^{4}$ \\ 'Department of Entomology, Faculty of Science, \\ Ain Shams University, Abbassiah, 11566, Cairo, Egypt
}

${ }^{2}$ Wuxi Centre for Disease Control and Prevention, Wuxi, Jiangsu 214023, China

${ }^{3}$ Current Address: Central Life Sciences, 12111 Ford Rd, Dallas, TX 75234

\begin{abstract}
${ }^{4}$ Anastasia Mosquito Control District, 120 EOC Drive, St. Augustine, FL 32092, U.S.A.
\end{abstract}

\author{
Guest Editor: Steve Peper
}

\begin{abstract}
Spatial repellents (SR), include pyrethroid insecticides that are highly volatile at low temperatures and with high lethal activities against mosquitoes, mainly Aedes vectors of arboviral diseases. Of these SR, metofluthrin is widely used in various devices for repellent consumer products. This article reports the susceptibility status of $A e$. aegypti Puerto Rico permethrin-resistant laboratory strain (PR) and Orlando susceptible laboratory strain (ORL) to metofluthrin and permethrin using the CDC glass bottle bioassay. The time-mortality relationships showed that the permethrin-resistant PR strain is highly resistant to both permethrin and metofluthrin compared to the susceptible ORL strain. The resistant ratio (RR) based on the killing time (KT) $\left(\mathrm{KT}_{50}-\mathrm{PR} / \mathrm{KT}_{50}-\mathrm{ORL}\right)$ was 30 - and 5- folds for permethrin and metofluthrin, respectively. The results also showed that the PR strain is less resistance to metofluthrin than to permethrin, with a three-fold $\mathrm{RR}\left(\mathrm{KT}_{50}-\mathrm{PR}\right.$-per $\left./ \mathrm{KT}_{50}-\mathrm{PR}-\mathrm{met}\right)$. These results indicate the potential risk of developing cross-resistance of metofluthrin in permethrin-resistant mosquitoes. Integrated vector management in mosquito control should be considerate of how consumer products and field operations interact to accelerate cross resistance to pyrethroids.
\end{abstract}

Key Words: Aedes aegypti, resistance, metofluthrin, permethrin, spatial repellents

\section{INTRODUCTION}

Aedes aegypti (Linn.) is a major vector of arboviral disease causing pathogens, such as yellow fever, dengue, zika, and chikungunya viruses. Application of repellents is one technique for prevention and control of mosquitoes and mosquito-borne diseases. Spatial repellents (SR) include a class of volatile pyrethroids, such as transfluthrin and metofluthrin, which have high insecticidal activities (Bibbs et al. 2018), high volatility at low temperatures, and low mammalian toxicity (Sugano and Ishiwatari 2012). These features facilitated their use in different commercial mosquito repellent devices (coils, liquid, cold fan vaporizers, paper and resinsbased emanators) against various mosquito vectors, mainly, Ae. aegypti and Ae. albopictus Skuse (Ujihara et al. 2004, Lucas et al. 2007, Sugano and Ishiwatari 2012). Paper-based emanators / devices of metofluthrin were highly effective against Aedes and Ochlerotatus species with up to $90 \%$ reduction in hostlanding rate (Lucas et al. 2007, Xue et al. 2012). In experimental rooms, metofluthrin resin-based emanators completely inhibited host-seeking and host-biting behaviors of $A e$. aegypti laboratory colony, and caused rapid knockdown (KD) and mortality (90\% in $<1 \mathrm{~h} \mathrm{hr}$ ). Using the resin emanators, repellent efficacy lasted for 20 days, which indi- 
cates their possible use to replace insecticidal indoor residual spraying (Ritchie and Devine 2013). The repellent activity of the metofluthrin-based OFF! Clip-on device was tested in semi-field conditions at different distances, and the results showed that metofluthrin was highly effective (up to $100 \%$ $\mathrm{KD}$ and mortality) against $A e$. aegypti when exposed for $60 \mathrm{~min}$ at $0.3 \mathrm{~m}$ from the device (Bibbs and Xue 2015). In glass chamber experiments on different mosquito pyrethroidbased coils in Malaysia, metofluthrin was the most effective against Ae. albopictus among the compared pyrethroids in regards to $\mathrm{KD}$ and killing time (KT). There was considerable variation in mortalities $(5-100 \%)$ and $\mathrm{KT}_{50}$ (2.5-17 $\mathrm{min}$ ), suggesting the presence of cross-resistance between the tested pyrethroids (Chen et al. 2018). Metofluthrin impregnated nets inconsistently repelled $A e$. aegypti and Anopheles dirus in Thailand based on spatial activity indices measured from sentinel and cone bioassays (Ponlawat et al. 2017). Metofluthrin emanators were moderately effective in reducing the landing rate and KD levels of Ae. aegypti (Darbro et al. 2017). Similarly, in Cambodia, slow-release metofluthrin emanators caused variable (47$67 \%$ ) reduction of landing rates of Anopheles species collected in tents or by CDC traps deployed in test premises (Charlwood et al. 2016). Metofluthrin affects host-seeking and biting behavior through a cascade of sequential events of agitation, confusion and knockdown of the target mosquitoes (Buhagiar et al. 2017a). However, prolonged exposure of the mosquito to sub-optimum doses of the active ingredient, especially at the margins of the chemical or device effective range "harbourage area", results in variable responses and the subsequent development of resistance (Buhagiar et al. 2017b). In a cage bioassay mosquitoes exposed to metofluthrin (10\% a.i.) for $60 \mathrm{~min}$ resulted in reduced mortality in Ae. aegypti females, with considerable mortality in males after 40 min. There were no obvious effects on the surviving females' fecundity (Buhagiar et al. 2017b). In Mexico, variable mortalities (41$100 \%$ ) were reported in Ae. aegypti exposed to 13 types of aerosolized insecticides com- monly used in houses, due to variable insecticide type, concentration, formulation, and method of application or spraying (KuriMorales et al. 2018). In another field study in Mexico, up to $50 \%$ reduction in mortality was observed in Ae. aegypti susceptible and field resistant strains exposed to aerosol insecticides, plug-in and coil devices, with significant increase in the frequency of $\mathrm{KD}$ resistant homozygous allele, $k d r$ I1016 (Gray et al. 2018). Widespread pyrethroid resistance in Ae. aegypti further confounds effective domestic mosquito management (Estep et al. 2017). Such findings highlight the risks of increased use of house-hold insecticides and repellents for personal protection. New pyrethroid formulations could contain synergists such as piperonyl butoxide or alternatives to enhance insecticide efficacy; or formulations could pair multiple active ingredients, particularly those that mosquitoes do not share resistance between (Bingham et al. 2011, Gray et al. 2018). It is equally important to educate consumers for well-informed use of over-the-counter insecticides and repellent devices to emphasize the role of citizens in integrated management and control programs (Vasques-Prokopec et al. 2017). In an attempt to generate useful information for the aforementioned goals, we report the evaluation of the spatial repellent compound-metofluthrin using the CDC bottle bioassay against susceptible and resistant Ae. aegypti strains.

\section{MATERIALS AND METHODS}

Female mosquitoes from a pyrethroid susceptible laboratory colony of Ae. aegypti 1952 Orlando (ORL) strain were provided by the United States Department of Agriculture, Center for Medical, Agricultural, and Veterinary Entomology (USDA-CMAVE), Gainesville, FL. This colony was reared at insectary facilities of the Anastasia Mosquito Control District (AMCD) in St. Augustine, Florida. Mosquitoes were maintained at $26 \pm$ $1.0^{\circ} \mathrm{C}, 65-80 \%$ relative humidity, and a photoperiod of 14:10 hr (L:D). The adult mosquitoes were provided $10 \%$ sugar solution ad libitum. For comparison, pyrethroid resistant 
Ae. aegypti Puerto Rico (PR) strain was obtained from the USDA-CMAVE. Mosquitoes used were 19 generations of permethrinresistance and reared under conditions as described in Pridgeon et al. (2008). Briefly, the mosquito eggs were allowed to hatch in a flask and remained overnight until transfer to a plastic tray containing distilled water with larval food. Mosquitoes were reared in an environmentally-controlled chambers set with a temperature profile representing a simulated summer day regimen (ranging from 22 to $30^{\circ} \mathrm{C}$ ) and $80 \% \mathrm{RH}$. Incandescent lighting was set to a crepuscular profile with a photoperiod of $14 \mathrm{hr}: 10 \mathrm{hr}$ (L:D), including $2 \mathrm{hr}$ of simulated dawn and $2 \mathrm{hr}$ of simulated dusk. Adults were held in a screened cage and provided 10\% sucrose ad libitum. All mosquitoes selected for testing were 5-8 d old, non-blood-fed females. Technical grade $98.1 \%$ permethrin (45614 Pestanal, Sigma-Aldrich Co. LLC, St. Louis, MO) and $95.6 \%$ metofluthrin (SumiOne, Sumitomo Chemical Company Ltd., Tokyo, Japan) insecticides were dissolved in $1 \mathrm{ml}$ of acetone to treat $250 \mathrm{ml}$ glass bottles according to procedures outlined in Brogdon and McAllister (1998). The final concentration was $43 \mathrm{ng}$ / bottle for each insecticide. Each bottle was filled with 15-20 Ae. aegypti adults and then observed for a maximum of 120 min. For each replicate, three bottles were assigned to ORL strain, three bottles were assigned to PR strain, and one bottle each was assigned to a control per insecticide. A total of 16 bottles were used each time. Permethrin and metofluthrin were replicated three times on different dates. The mean number of dead/moribund mosquitoes in bottles was plotted over time for each insecticide and the control. The time-mortality relationship lines were plotted and the median lethal KT values were deduced from the lines for each strain and each insecticide tested. For comparison of efficacies and susceptibility level of each strain, the resistance ratios (RR) were calculated by dividing the KT-50 values of the resistant strain by the susceptible strain. Abbott formula was not performed due to almost zero number of mortality in control group.

\section{RESULTS AND DISCUSSION}

Insecticide susceptibility and resistance of both permethrin and metofluthrin were detected in the PR strain as compared to the ORL strain (Fig. 1 and Table 1). The RR $\left(\mathrm{KT}_{50}-\mathrm{PR} / \mathrm{KT}_{50}-\mathrm{ORL}\right)$ was 30 and 15 folds for permethrin and metofluthrin, respectively.

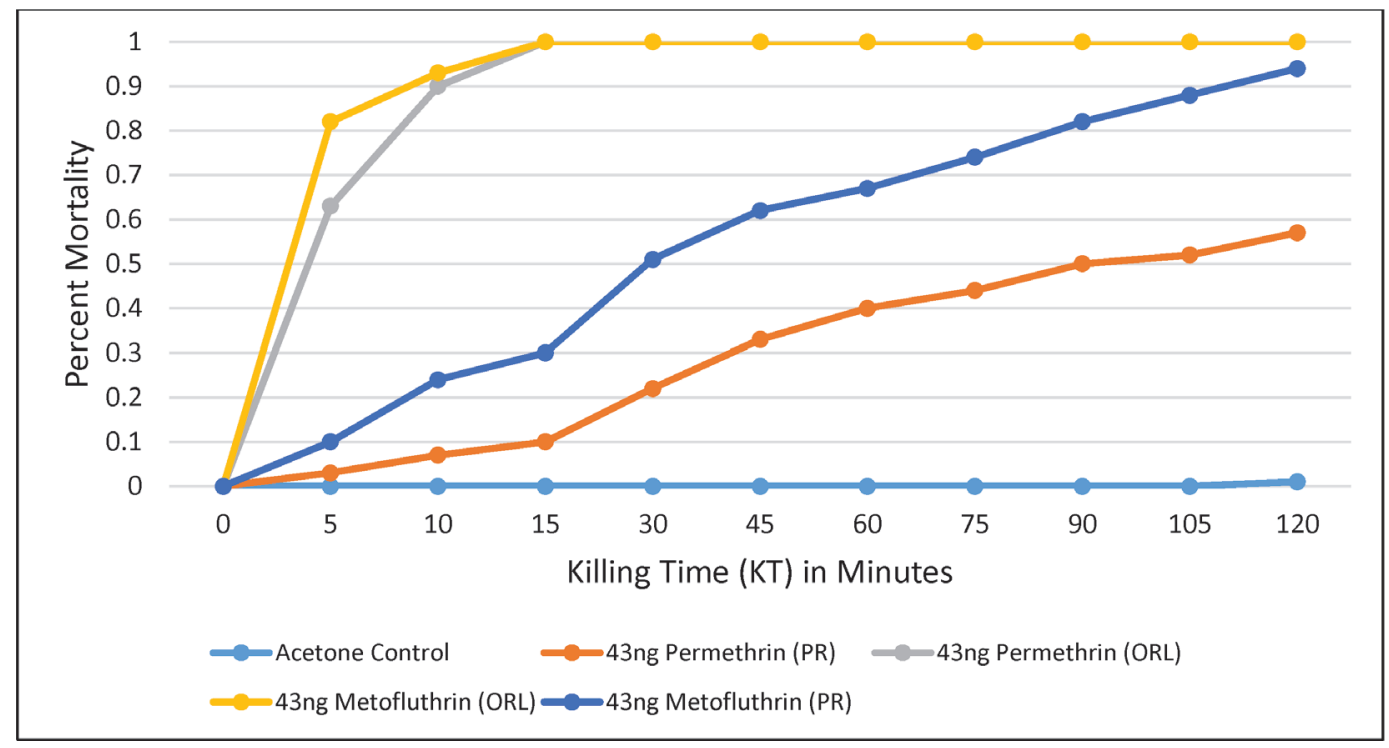

Figure 1. The time-mortality relationships for Orlando susceptible lab (ORL) and Puerto Rico resistant lab (PR) strains of Aedes aegypti exposed to permethrin and metofluthrin using the CDC bottle insecticide bioassay. 
The PR strain was less resistant to metofluthrin than to permethrin, with $\mathrm{RR}\left(\mathrm{KT}_{50}-\mathrm{PR}-\right.$ $\mathrm{per} / \mathrm{KT}_{50}$-PR-met) of three-fold. However, it is clear that substantial cross resistance is present in the permethrin-resistant mosquitoes. Exposure of the PR strain to permethrin for 120 min caused only $57 \%$ mortality, while metofluthrin caused $94 \%$. The susceptible ORL strain had comparable susceptibility to both permethrin and metofluthrin, where $\mathrm{KT}_{100}$ was $<15 \mathrm{~min}$. In this report, the permethrin-resistant Ae. aegypti PR strain showed moderate level of cross-resistance to metofluthrin, with RR of 30- fold and 15-fold for permethrin and metofluthrin, respectively. This result is similar with results from lab and field experiments reported by Chen et al. (2018), Kuri-Morales et al. (2018), and Gray et al. (2018). Observed reductions in susceptibility are due to the long-term and inconsistent exposure of the mosquitoes to sub-lethal doses of the insecticide active ingredients used in different formulations, whether through space spraying, residual spraying, or vapor repellents (Kuri-Morales et al. 2018, Gray et al. 2018). In the State of Florida, there was wide-spread $k d r$ resistance in Ae. aegypti field strains (RR of 6-61 fold to permethrin) with significant increase in the frequencies of homozygous and heterozygous $k d r$ alleles of V1016I and F1534C (Estep et al. 2018). In contrast, in the same study, lower levels of resistance were reported in Ae. albopictus strains, even though they were collected from the same geographies that contained resistant Ae. aegypti strains. These various studies show the complex nature of $k d r$ resistant dynamics and inheritance in different mosquito species or strains (Ae. aegypti and Ae. albopictus) and the potential spread of cross-resistance between different pyrethroids commonly used for vector control. Therefore, for better monitoring and management of resistance at early stages, it is essential to measure the susceptibility levels of various mosquito populations using a combination of bioassays (WHO, CDC bottle, and topical application bioassays) and molecular assays (genotyping and allele frequency) (Reid et al. 2014, Al-Nazawi et al. 2017, Estep et al. 2017, 2018). Chemical 
adulticides are still the main means of controlling mosquito vectors of diseases. Whether by aerosol or repellents, pyrethroids are the most used insecticide as spatial insect repellent compounds for personal protection against the bites of adult mosquitoes in domestic and area-wide scenarios. Spatial repellent volatile pyrethroids are highly insecticidal, and their use to control mosquitoes is increasing. The combined pressures from pyrethroids deployed in home use, commercial agriculture, and vector control has resulted in development of $k d r$ in response to almost all pyrethroid active ingredients (Wagman et al. 2015, Estep et al. 2018, Gray et al. 2018). Sustainable mosquito control programs hinge on routine monitoring of insecticide efficacy. This can be performed through both conventional insecticide susceptibility bioassays (the WHO and CDC bioassays) and molecular genotyping of resistance genes such as $k d r$ alleles (Estep et al. 2018). Our findings show that the permethrin-resistant PR strain is highly resistance to both permethrin and metofluthrin compared to the susceptible ORL strain, and the PR strain is less resistance to metofluthrin than to permethrin.

\section{ACKNOWLEDGEMENTS}

We would like to thank McLoughlin Gormley King (MGK) Company and Sumitomo Chemical Company for providing technical grade metofluthrin and partial fund and USDA- CMAVE/Mosquito and Fly Unit (JJ Becnel's laboratory), Gainesville, FL for providing the resistant strain of Ae. aegypti for this study. The Anastasia Mosquito Control District does not endorse specific commercial products. Mention of propriety goods are for research purposes only.

\section{REFERENCES CITED}

Al-Nazawi AM, Aqili J, Alzahrani M, McCall PJ, Weetman D. 2017. Combined target site (kdr) mutations play a primary role in highly pyrethroid resistant phenotypes of Aedes aegypti from Saudi Arabia. Parasit Vectors 10:161.

Bibbs CS, Xue RD. 2015. Off! Repellent devices with metofluthrin tested on Aedes aegypti (Diptera: Cu- licidae) for mortality at different time intervals and distance. J Med Entomol. 53:480-483.

Bibbs CS, Tsikolia M, Bernier UR, Bloomquist JB, Xue RD, Kaufman PE. 2018. Vapor toxicity of five volatile pyrethroids against Aedes aegypti, Ae. albopictus, Culex quinquefasciatus, and Anopheles quadrimaculatus (Diptera: Culicidae). Pest Management Science 74:2699-2706.

Bingham G, Strode C, Tran L, Khoa PT, Jamet HP. 2011. Can piperonyl butoxide enhance the efficacy of pyrethroids against pyrethroid-resistant Aedes aegypti? Trop Med Int Health 16:492-500.

Brogdon WG McAllister JC. 1998. Simplification of adult mosquito bioassays through use of time-mortality determinations in glass bottles. J Am Mosq Control Assoc. 14:159-164.

Buhagiar TS, Devine GL, Ritchie SA. 2017a. Metofluthrin investigations into the use of a volatile spatial pyrethroid in a global spread of dengue, chikungunya and zika viruses. Parasit Vectors 10:270.

Buhagiar TS, Devine GJ, Ritchie SA. 2017b. Effects of sublethal exposure to metofluthrin on the fitness of Aedes aegypti in a domestic setting in Cairns, Queensland. Parasit Vectors. 10:274.

Chen CD, Chin AC, Lau KW, Low VL, Lee HL, Lee PKY, Azidah AA, Sofian-Azirun M. 2018. Bioefficacy evaluation of commercial mosquito coils containing metofluthrin, d-allethrin, d-trans allethrin, and prallethrin against Aedes albopictus (Diptera: Culicidae) in Malaysia. J Med Entomol. 55:1651-1655.

Charlwood JD, Nenhep S, Protopopoff N, Sovannaroth S, Morgan JC, Hemingway J. 2016. Effects of the spatial repellent metofluthrin on landing rates of outdoor biting anophelines in Cambodia, Southeast Asia. Med Vet Entomol. 30:229-234.

Darbro JM, Muzari MO, Giblin A, Adamczyk RM, Ritchie SA, Devine GJ. 2017. Reducing biting rates of Aedes aegypti with metofluthrin: investigations in time and space. Parasit Vectors. 10: 69.

Estep AS, Sanscrainte ND, Waits CM, Bernard SJ, Lloyd AM, Lucas KJ, Buckner EA, Vaidyanathan R, Morreale R, Conti LA, Becnel JJ. 2018. Quantification of permethrin resistance and kdr alleles in Florida strains of Aedes aegypti (L.) and Aedes albopictus (Skuse). PLoS Negl Trop Dis. 12:e0006544.

Estep AS, Sanscrainte ND, Waits CM, Louton JE, Becnel JJ. 2017. Resistance Status and Resistance Mechanisms in a Strain of Aedes aegypti (Diptera: Culicidae) From Puerto Rico. J Med Entomol. 54:1643-1648.

Gray L, Florez SD, Barreiro AM, Vadillo-Sánchez J, González-Olvera G, Lenhart A, Manrique-Saide P, Vazquez-Prokopec GM. 2018. Experimental evaluation of the impact of household aerosolized insecticides on pyrethroid resistant Aedes aegypti. Sci Rep. $8: 12535$.

Kuri-Morales PA, Correa-Morales F, González-Acosta C, Moreno-Garcia M, Dávalos-Becerril E, Benitez-Alva JI, Peralta-Rodriguez J, Salazar-Bueyes V, GonzálezRoldán JF. 2018. Efficacy of 13 commercial household aerosol insecticides against Aedes aegypti (Diptera: Culicidae) from Morelos, Mexico. J Med Entomol. 55:417-422.

Lucas JR, Shono Y, Iwasaki T, Ishiwatari T, Spero N, Benzon G. 2007. US laboratory and field trials of metofluthrin (SumiOne ${ }^{\circledR}$ ) emanators for reducing mosquito biting outdoors. J Am Mosq Control Assoc. 23:47-54.

Ponlawat A, Harwood JF, Putnam JL, Nitatsukprasert C, Pongsiri A, Kijchalao U, Linthicum KJ, Kline DL, 
Clark GG, Obenauer PJ, Doud CW, Mccardle PW, Richardson AG, Szumlas DE, Richardson JH. 2017. Field evaluation of indoor thermal fog and ultra-low volume applications for control of Aedes aegypti in Thailand. J Am Mosq Control Assoc. 33:116-127.

Pridgeon JW, Pereira RM, Becnel JJ, Allan SA, Clark GG, Linthicum KJ. 2008. Susceptibility of Aedes aegypti, Culex quinquefasciatus Say, and Anopheles quadrimaculatus Say to 19 pesticides with different modes of action. J Med Entomol. 45:82-87.

Reid WR, Thornton A, Pridgeon JW, Becnel JJ, Tang F, Estep A, Clark GG, Allan SA, Liu N. 2014. Transcriptional analysis of four family 4 P450s in a Puerto Rico strain of Aedes aegypti (Diptera: Culicidae) compared with an Orlando strain and their possible functional roles in permethrin resistance. J Med Entomol. 51:605-615.

Ritchie SA, Devine GJ. 2013. Confusion, knock-down and kill of Aedes aegypti using metofluthrin in domestic settings: A powerful tool to prevent dengue transmission? Parasit Vectors 6: 262.

Sugano M, Ishiwatari T. 2012. The biological activity of a novel pyrethroid: metofluthrin. Top Curr Chem. 314: 203-220.
Ujihara K, Mori T, Iwasaki T, Sugano M, Shono Y, Matsuo N. 2004. Metofluthrin: a potent new synthetic pyrethroid with high vapor activity against mosquitoes. Biosci Biotechnol Biochem. 68:170-174.

Vazquez-Prokopec GM, Medina-Barreiro A, CheMendoza A, Dzul-Manzanilla F, Correa-Morales F, Guillermo-May G, Bibiano-Marín W, Uc-Puc V, Geded-Moreno E, Vadillo-Sánchez J, Palacio-Vargas J, Ritchie SA, Lenhart A, Manrique-Saide P. 2017. Deltamethrin resistance in Aedes aegypti results in treatment failure in Merida, Mexico. PLoS Negl Trop Dis. 11(6):e0005656.

Wagman JM, Achee NL, Grieco JP. 2015. Insensitivity to the spatial repellent action of transfluthrin in Aedes aegypti: a heritable trait associated with decreased insecticide susceptibility. PLoS Negl Trop Dis. 9:e0003726.

Xue RD, Qualls WA, Smith ML, Weaver JR, Gaines MK, Debboune M. 2012. Field evaluation of the OFF! Clip-on mosquito repellent (Metofluthrin) against Aedes albopictus and Aedes taeniorhynchus (Diptera: Culicidae) in Northeastern Florida. J Med Entomol. 49:652-655. 